\title{
The breakup length of harmonically stimulated capillary jets
}

F. J. García, H. González, J. R. Castrejón-Pita, and A. A. Castrejón-Pita

Citation: Appl. Phys. Lett. 105, 094104 (2014); doi: 10.1063/1.4894576

View online: https://doi.org/10.1063/1.4894576

View Table of Contents: http://aip.scitation.org/toc/apl/105/9

Published by the American Institute of Physics

\section{Articles you may be interested in}

Breakup length of forced liquid jets

Physics of Fluids 15, 2469 (2003); 10.1063/1.1593023

Dynamic nozzles for drop generators

Review of Scientific Instruments 86, 115101 (2015); 10.1063/1.4934811

On the breakup of viscous liquid threads

Physics of Fluids 7, 1529 (1995); 10.1063/1.868540

Comment on "Breakup length of forced liquid jets" [Phys. Fluids 15, 2469 (2003)]

Physics of Fluids 18, 019101 (2006); 10.1063/1.2159447

Drop formation from a capillary tube: Comparison of one-dimensional and two-dimensional analyses and occurrence of satellite drops

Physics of Fluids 14, 2606 (2002); 10.1063/1.1485077

Breakup of capillary jets with different disturbances

Physics of Fluids 28, 012101 (2016); 10.1063/1.4938095

\section{Instruments for Advanced Science}

Contact Hiden Analytical for further details: w www.HidenAnalytical.com E info@hiden.co.uk CLICK TO VIEW our product catalogue

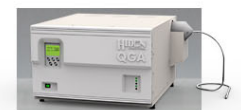

Gas Analysis dynamic measurement of reaction gasstreams cataysis and thermal analysis molecular beam studes dissolved species probes fermentation, envirormental and ecological stud

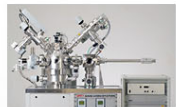

Surface Science UHVTPD

, end point detection in in beam etch , elemental imaging-surface mapping

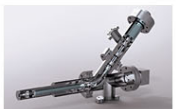

Plasma Diagnostics , plasma source characterization etch and depositon process reaction kinetic studies

analysis of neutral and radical species

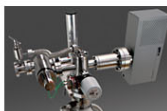

Vacuum Analysis partial pressure measurement and control of process gases reactive sputter process control vacuum diagnostic 


\title{
The breakup length of harmonically stimulated capillary jets
}

\author{
F. J. García, ${ }^{1}$ H. González, ${ }^{2, a)}$ J. R. Castrejón-Pita, ${ }^{3}$ and A. A. Castrejón-Pita ${ }^{4}$ \\ ${ }^{1}$ Departamento de Física Aplicada I, Escuela Politécnica Superior, Universidad de Sevilla, \\ c/Virgen de África, 7. 41011-Sevilla, Spain \\ ${ }^{2}$ Departamento de Física Aplicada III, Escuela Técnica Superior de Ingenieros, Universidad de Sevilla, \\ Camino de los Descubrimientos, s/n 41092-Sevilla, Spain \\ ${ }^{3}$ Department of Engineering, University of Cambridge, 17 Charles Babbage Road, Cambridge CB3 OFS, \\ United Kingdom \\ ${ }^{4}$ Department of Engineering Science, University of Oxford, Parks Road, Oxford OX1 3PJ, United Kingdom
}

(Received 20 May 2014; accepted 20 August 2014; published online 5 September 2014)

\begin{abstract}
A simple transfer function that can predict the breakup length of a pressure-modulated capillary jet is rigorously deduced from first principles. In this paper, the initial velocity modulation of a stimulated jet is given in terms of its pressure amplitude by means of a generalized Bernoulli equation, which in turn is connected to the breakup time through a two-mode linear analysis. The predicted breakup length is compared against experimental results with water jets emerging from a thin 1 mm-diameter orifice for different pressure modulations. These experiments agree better with the presented theoretical prediction than with a previously established model. (C) 2014 AIP Publishing LLC.
\end{abstract}

[http://dx.doi.org/10.1063/1.4894576]

The breakup of liquid jets into droplets due to the capillary instability has been the focus of rigorous studies for more than two centuries. ${ }^{1}$ The possibility of generating monodisperse microdroplets has increased the attention to this subject beyond its pure scientific interest. ${ }^{2}$ Some modern technologies, such as $2 \mathrm{D}$ and $3 \mathrm{D}$ inkjet printing in continuous-jet mode $(\mathrm{CIJ}){ }^{3}$ rely on this breakup process, and fundamental research is needed if the technology is to advance and overcome its current limits. In particular, the prediction of the breakup length under controlled stimulation by an electromagnetic or piezoelectric actuator is often required. Practitioners have previously dealt with empirical transfer functions that relate stimulation and breakup lengths without having the knowledge of the role of the relevant parameters. The problem is not so much the behavior of the actuator as the link between the hydrodynamics of chamber from which the jet emerges, the exit orifice and the jet itself. The present work provides such a theory to address this, which is validated with original experiments.

Consequently, the system under consideration is composed by a pressurized chamber, a small round exit orifice and an emerging capillary jet. Assuming that the liquid is incompressible, the Navier-Stokes equation can be conveniently written as

$$
\rho \frac{\partial \mathbf{v}}{\partial t}+\nabla\left(p+\frac{1}{2} \rho v^{2}+\rho g z\right)-\rho \mathbf{v} \times \nabla \times \mathbf{v}-\mu \nabla^{2} \mathbf{v}=0
$$

where $\rho$ is the density of the liquid, $\mu$ is the dynamic viscosity, $p$ and $\mathbf{v}$ are the pressure and velocity (with modulus $v$ ) as functions of position $\mathbf{r}$ and time $t ; g$ is the gravitational acceleration, and $z$ is the vertical coordinate. Integrating (1) along the instantaneous streamline $\Gamma(t)$ connecting any inner point of the chamber (labeled with subscript "c") and a point of

\footnotetext{
a)helio@us.es
}

the emerged jet after the relaxation of its velocity profile (subscript "j") yields

$$
\begin{aligned}
& \rho \int_{\Gamma} \mathrm{d} \mathbf{r} \cdot \frac{\partial \mathbf{v}}{\partial t}+p_{\mathrm{j}}-p_{\mathrm{c}}+\frac{1}{2} \rho\left(v_{\mathrm{j}}^{2}-v_{\mathrm{c}}^{2}\right) \\
& \quad+\rho g\left(z_{\mathrm{j}}-z_{\mathrm{c}}\right)+\Delta p_{\mathrm{visc}}=0
\end{aligned}
$$

where the third term in the left-hand side of (1) gives no contribution and $\Delta p_{\text {visc }} \equiv-\mu \int_{\Gamma} \mathrm{d} \mathbf{r} \cdot \nabla^{2} \mathbf{v}$ is the pressure drop along the streamline due to viscous losses. Equation (2) is an extended Bernoulli's equation including an unsteady inertial term and viscous losses. Standard textbooks in fluid mechanics (see, for instance, Ref. 4) derive, as a rule, Bernoulli's equation for more restrictive cases, but the interested reader can find in Ref. 5 a very general version, deduced in the same way that Equation (2), which is a special case of it.

Some simplifications are in order:

(i) The dynamic pressure at the chamber, $\frac{1}{2} \rho v_{\mathrm{c}}^{2}$, is usually negligible compared to that at the jet due to mass conservation and the difference in cross sectional areas at these points.

(ii) The unsteady inertial term can be evaluated under the assumption of plug profile to 4

$$
\rho \int_{\Gamma} \mathrm{d} \mathbf{r} \cdot \frac{\partial \mathbf{v}}{\partial t}=\rho l_{\mathrm{i}} \frac{\mathrm{d} v_{\mathrm{o}}}{\mathrm{d} t}=c_{\mathrm{c}} \rho l_{\mathrm{i}} \frac{\mathrm{d} v_{\mathrm{j}}}{\mathrm{d} t},
$$

where $v_{\mathrm{o}}$ is the mean velocity at the exit orifice and $l_{\mathrm{i}}$ is an inertial length, roughly equal to the exit orifice length $l_{\mathrm{o}}$. The inertial length can be redefined to account for the contribution of the regions adjacent to the exit orifice. In Eq. (3), the contraction coefficient $c_{\mathrm{c}} \equiv v_{\mathrm{o}} / v_{\mathrm{j}}=D_{\mathrm{j}}^{2} / D_{\mathrm{o}}^{2}$ was used, with $D_{\mathrm{j}}$ the jet diameter after relaxation of the velocity profile and $D_{\mathrm{o}}$ is the exit orifice diameter. The diameter ratio was studied experimentally in Ref. 6. 
(iii) Finally, the viscous losses can be estimated following the classic work of Langhaar ${ }^{7}$ as:

$$
\Delta p_{\mathrm{visc}}=\left(\frac{64 l_{\mathrm{o}}}{\operatorname{Re}_{\mathrm{o}} D_{\mathrm{o}}}+K\right) \frac{1}{2} \rho v_{\mathrm{o}}^{2}-\frac{1}{2} \rho v_{\mathrm{j}}^{2},
$$

where the Reynolds number at the exit orifice has been defined as $\operatorname{Re}_{\mathrm{o}} \equiv \rho v_{\mathrm{o}} D_{\mathrm{o}} / \mu$ and $K$ is a dimensionless constant usually called entrance correction factor, for which several theoretical and experimental investigations report values lying in the range $2.11<K<2.45$ (Ref. 8). Equation (4) does not include the viscous losses during the relaxation of the jet velocity profile, and is a good approximation only when $l_{\mathrm{o}} / D_{\mathrm{o}} \mathrm{Re}_{\mathrm{o}} \gg 1$, i.e., for fully developed Hagen-Poiseuille velocity profiles. If that is not the case, (4) seems to correctly describe the viscous losses with empirical values for $K$ and $l_{0} .{ }^{9}$

Given the previous assumptions, and writing $v_{0}$ in terms of $v_{\mathrm{j}}$, Eq. (2) yields

$$
p_{\text {eff }}=c_{\mathrm{c}}^{2} K \frac{1}{2} \rho v_{\mathrm{j}}^{2}+c_{\mathrm{c}} \rho l_{\mathrm{i}} \frac{\mathrm{d} v_{\mathrm{j}}}{\mathrm{d} t}+c_{\mathrm{c}} \frac{32 \mu l_{\mathrm{o}}}{D_{\mathrm{o}}^{2}} v_{\mathrm{j}},
$$

where the effective pressure is defined as $p_{\text {eff }} \equiv p_{\mathrm{c}}+$ $\rho g\left(z_{\mathrm{c}}-z_{\mathrm{j}}\right)-2 \gamma / D_{\mathrm{j}}$ and $\gamma$ is the surface tension. Equation (5) directly relates the pressure in a given point in the chamber to the jet velocity in the region where the profile has relaxed to a plug one. In the steady basic flow, the jet velocity, denoted as $v_{0}$, is obtained from the steady effective pressure $p_{0}$, by solving

$$
p_{0}=c_{\mathrm{c}}^{2} K \frac{1}{2} \rho v_{0}^{2}+c_{\mathrm{c}} \frac{32 \mu l_{\mathrm{o}}}{D_{\mathrm{o}}^{2}} v_{0} .
$$

The actuator modulates the pressure inside the chamber harmonically. Therefore, the effective pressure reads

$$
p_{\text {eff }}(t)=p_{0}+p_{1} \cos (\omega t)=p_{0}+\frac{1}{2} p_{1} \mathrm{e}^{\mathrm{i} \omega t}+\frac{1}{2} p_{1} \mathrm{e}^{-\mathrm{i} \omega t},
$$

where the amplitude of the effective pressure $p_{1}$ is real and $\omega$ is the angular frequency of the modulation. This leads to a perturbed jet velocity that, after linearization, becomes

$$
v_{\mathrm{j}}(t)=v_{0}+\frac{1}{2} v_{1} \mathrm{e}^{\mathrm{i} \omega t}+\frac{1}{2} v_{1}^{*} \mathrm{e}^{-\mathrm{i} \omega t}+\cdots,
$$

where $v_{1}^{*}$ denotes the complex conjugate of the velocity amplitude. Scaling the velocity and pressures as $\breve{v}_{1} \equiv v_{1} / v_{0}$ and $\breve{p}_{0} \equiv p_{0} /\left(\frac{1}{2} \rho v_{0}^{2}\right), \breve{p}_{1} \equiv p_{1} /\left(\frac{1}{2} \rho v_{0}^{2}\right)$, Eqs. (5), (7), and (8) give

$$
\breve{v}_{1}=\frac{\breve{p}_{1}}{2 c_{\mathrm{c}}^{2} K+X_{\mathrm{r}}+\mathrm{i} X_{\mathrm{i}}},
$$

where $X_{\mathrm{r}}$ and $X_{\mathrm{i}}$ are dimensionless parameters

$$
X_{\mathrm{r}}=c_{\mathrm{c}}^{2} \frac{64}{\operatorname{Re}_{\mathrm{o}}} \frac{l_{\mathrm{o}}}{D_{\mathrm{o}}}, \quad X_{\mathrm{i}}=c_{\mathrm{c}} \frac{2 \omega l_{\mathrm{i}}}{v_{0}},
$$

playing the roles of real and imaginary parts of an acoustic impedance associated with the exit orifice. Physically, $X_{\mathrm{r}}$ accounts for the resistance due to viscous dissipation and $X_{\mathrm{i}}$ for the inertia of the liquid inside the exit orifice. Equation (6) now reads $\breve{p}_{0}=c_{\mathrm{c}}^{2} K+X_{\mathrm{r}}$.

The next step is to relate the perturbation of the jet velocity after the viscous relaxation with the breakup length. The jet evolution is governed by capillary forces, so it is advantageous to change the dimensionalization by scaling time with the capillary time $\left[\rho D_{\mathrm{j}}^{3} /(8 \gamma)\right]^{1 / 2}$. In the following, a bar over a symbol means "made dimensionless with the capillary scales." The new dimensionless velocity $\bar{v} \equiv v /\left[2 \gamma /\left(\rho D_{\mathrm{j}}\right)\right]^{1 / 2}$ is related to the previous one $\breve{v}$ by $\breve{v} \equiv v / v_{0}=\bar{v} / \mathrm{We}^{1 / 2}$, where $\mathrm{We} \equiv \rho D_{\mathrm{j}} v_{0}^{2} / 2 \gamma$ is the Weber number.

Provided that the Weber number is much greater than unity, the temporal stability analysis applies: ${ }^{10,11}$ from a reference frame moving with the mean flow, the jet is periodically perturbed in the longitudinal coordinate with associated dimensionless wavenumber $\bar{k} \equiv D_{\mathrm{j}} \omega / 2 v_{0}$. As described in Ref. 12, the perturbation amplitudes of both the jet shape and velocity are related to the amplitudes of the dominant (growing for $\bar{k}<1$ ) and subdominant (decaying) capillary modes. Other decaying modes, the hydrodynamic modes, may be present but their influence can be disregarded. ${ }^{12}$ The boundary conditions at the nozzle must be translated into initial conditions for the temporal evolution of a periodic liquid column. As the contact line is fixed, the initial conditions must be purely impulsive, i.e., with velocity modulation and without initial deformation. The amplitudes of the dominant and subdominant capillary modes are given by

$$
\left|\bar{f}_{\mathrm{d}}\right|=-\left|\bar{f}_{\mathrm{s}}\right|=\frac{\frac{1}{2} \bar{k}}{\bar{\alpha}_{\mathrm{d}}-\bar{\alpha}_{\mathrm{s}}}\left|\bar{v}_{1}\right|,
$$

where $\bar{\alpha}_{\mathrm{d}} \equiv \bar{\alpha}_{\mathrm{d}}(\bar{k}, \mathrm{Oh})$ and $\bar{\alpha}_{\mathrm{s}} \equiv \bar{\alpha}_{\mathrm{s}}(\bar{k}, \mathrm{Oh})$ are the dimensionless dominant and subdominant growth rates, respectively, i.e., the relevant solutions of the classical Rayleigh's temporal dispersion relation, ${ }^{13,14}$ and $\mathrm{Oh} \equiv \mu /\left(\rho \gamma D_{\mathrm{j}} / 2\right)^{1 / 2}$ is the Ohnesorge number. Alternatively, approximate explicit expressions for these growth rates can be obtained by means of one-dimensional models, ${ }^{15}$ among which the averaged model gives the quadratic dispersion relation

$$
\bar{\alpha}^{2}\left(1+\bar{k}^{2} / 8\right)+3 \mathrm{Oh} \bar{k}^{2} \bar{\alpha}+\left(\bar{k}^{2}-\bar{k}^{4}\right) / 2=0,
$$

whose two solutions have errors of less than $0.5 \% .{ }^{15}$

Taking into account the transfer function between the perturbation of the pressure in the chamber and the jet velocity after relaxation, given by (9), the amplitude of the two capillary modes are determined by

$$
\left|\bar{f}_{\mathrm{d}}\right|=-\left|\bar{f}_{\mathrm{s}}\right|=\sqrt{\mathrm{We}} \frac{\frac{1}{2} \bar{k}}{\bar{\alpha}_{\mathrm{d}}-\bar{\alpha}_{\mathrm{s}}} \frac{\left|\breve{p}_{1}\right|}{\sqrt{\left(2 c_{\mathrm{c}}^{2} K+X_{\mathrm{r}}\right)^{2}+X_{\mathrm{i}}^{2}}} .
$$

The subsequent evolution of the jet deformation is

$$
|\bar{f}(t)|=\left|\bar{f}_{\mathrm{d}}\right|\left[\exp \left(\bar{\alpha}_{\mathrm{d}} \bar{t}\right)-\exp \left(\bar{\alpha}_{\mathrm{s}} \bar{t}\right)\right] .
$$

The condition $\left|\bar{f}\left(\bar{t}_{\mathrm{b}}\right)\right|=1$ accurately determines the breakup time $\bar{t}_{\mathrm{b}}$ as long as the non-linear part of the evolution is short compared to $\bar{t}_{\mathrm{b}} \cdot{ }^{12,16}$ For sufficiently long jets, the contribution of the subdominant mode to the jet evolution can be 
ignored and the term of the second exponential in Eq. (14) can be dropped, giving explicitly

$$
\bar{t}_{\mathrm{b}}=-\frac{1}{\bar{\alpha}_{\mathrm{d}}} \log \left(\left|\bar{f}_{\mathrm{d}}\right|\right) \text {. }
$$

Multiplication by $\mathrm{We}^{1 / 2}$ (dimensionless velocity) and substitution of (13) gives the dimensionless breakup length

$$
\bar{L}_{\mathrm{b}}=-\frac{\sqrt{\mathrm{We}}}{\bar{\alpha}_{\mathrm{d}}} \log \left[\frac{\frac{1}{2} \bar{k} \sqrt{\mathrm{We}}\left|\breve{p}_{1}\right|}{\left(\bar{\alpha}_{\mathrm{d}}-\bar{\alpha}_{\mathrm{s}}\right) \sqrt{\left(2 c_{\mathrm{c}}^{2} K+X_{\mathrm{r}}\right)^{2}+X_{\mathrm{i}}^{2}}}\right] .
$$

The only previous transfer function relating these magnitudes is that of Orme,${ }^{17}$ based on the unphysical assumption that the modulation in pressure produces a modulation in the initial jet radius, rather than in the velocity. In our notation, Orme's description reads $\left|\bar{f}_{\mathrm{d}}\right|=\left(1+\breve{p}_{1}\right)^{1 / 4}-1$ for pressure perturbations of arbitrary size, and

$$
\left|\bar{f}_{\mathrm{d}}\right|=\frac{1}{4} \breve{p}_{1}
$$

for small stimulation amplitudes. Apart from the rigorous deduction of (13) from first principles, the major differences with (17) are: (i) the inclusion of inertia and viscous losses at the orifice, (ii) the dependence of the initial perturbations on the stimulation frequency through the wavenumber and growth factors, and most importantly (iii) the factor $\mathrm{We}^{1 / 2}$ in the argument of the logarithm. The latter results in a dominant capillary mode $\bar{f}_{\mathrm{d}} \propto v_{0}^{-1}$, while Orme's result (17) gives $\bar{f}_{\mathrm{d}} \propto v_{0}^{-2}$.

In order to validate this theory, the experimental work by Curry and Portig ${ }^{18}$ seems to be the best candidate. However, as it happens with other experiments not designed specifically for this comparison, some necessary parameters were not provided. Therefore, an experimental setup, sketched in Fig. 1 was developed in order to test (16). Pure tri-distilled water $\left(\mu=1 \mathrm{mPam}^{-1}, \quad \gamma=0.072 \mathrm{Nm}, \quad\right.$ and $\rho=997.6 \mathrm{~kg} / \mathrm{m}^{3}$ ) was pumped from an open reservoir towards a sealed chamber that was half-filled with air, designed to act both as a pressure buffer and to trap impurities and bubbles. The flow was then directed to a second chamber, with one end consisting of a flexible rubber membrane connected to a mechanical vibrator (LDS V201). This

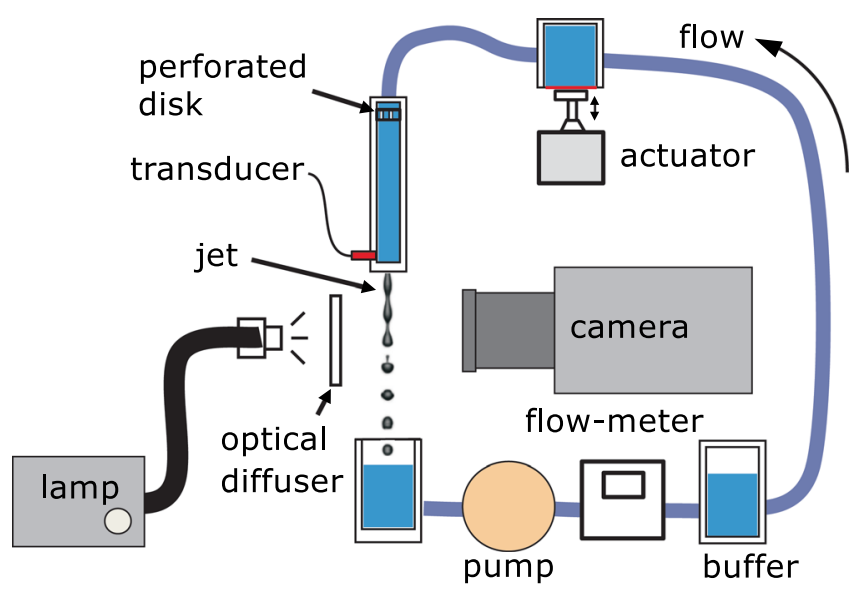

FIG. 1. Schematic diagram of the experimental setup. vibrator imposed the harmonic pressure perturbations to the fluid. The flow then continued to a $200 \mathrm{~mm}$ long cylindrical chamber $(50 \mathrm{~mm}$ in diameter), in which a $2 \mathrm{~mm}$-thick disk, with randomly positioned orifices, was placed close to the inlet to suppress any large entry vortices and to help induce the rapid development of the flow. The nozzle, a circular orifice of $1.006 \pm 0.003 \mathrm{~mm}$ in diameter, was located at the opposite end of this chamber and made of a $100 \pm 5 \mu \mathrm{m}$ thick brass shim.

A high-resolution temperature-compensated pressure sensor (Honeywell 40PC006G) was located on the side of the cylindrical chamber, $12 \mathrm{~mm}$ away from the base of the nozzle plate. This transducer monitored the static pressure and the amplitude of its sinusoidal perturbation close to the exit point. The reservoir, the pump, the chambers, and all instruments were mounted on different breadboard tables in order to avoid mechanical vibrations affecting the jet breakup. Average values of $p_{0}$ and $p_{1}$ were then extracted from data acquired over $3 \times 10^{3}$ complete oscillations for each experiment. The jet was visualized and the breakup length was measured by means of a standard shadowgraphy system coupled to a high-speed camera (Phantom V711). An acquisition rate of $50000 \mathrm{fps}$ and exposure time of $2 \mu$ sere used for all the experiments. Average breakup lengths, $L_{\mathrm{b}}$, were obtained by analysing a minimum of 20 breakup events for each value of $p_{1}$. A perturbation frequency of $680.0 \pm 0.5 \mathrm{~Hz}$, i.e., close to maximum growth rate conditions, was kept throughout all the experiments. An assemblage of representative images for different pressure amplitudes is shown in Fig. 2. The velocity of the jet was measured by two independent methods: (i) by measuring the distance between two consecutive main drops and relating it to the jet velocity as proposed in Refs. 19 and 20 or (ii) by generating a single drop in the middle of the jet by means of a single pressure pulse and recording its position as a function of time-its velocity and acceleration were then calculated by means of image analysis. In both cases, the initial velocity and acceleration are $2.72 \pm 0.02 \mathrm{~m} / \mathrm{s}$ and $9.1 \pm 0.1 \mathrm{~m} / \mathrm{s}^{2}$, respectively. This leads to deviations from the mean velocity (within the travel distances of interest) of less than 5\%. These small differences suggest, as expected, a small effect of gravity and the surrounding air on the jet evolution. From the flow rate, fixed to $1.725 \times 10^{-6} \mathrm{~m}^{3} / \mathrm{s}$, a

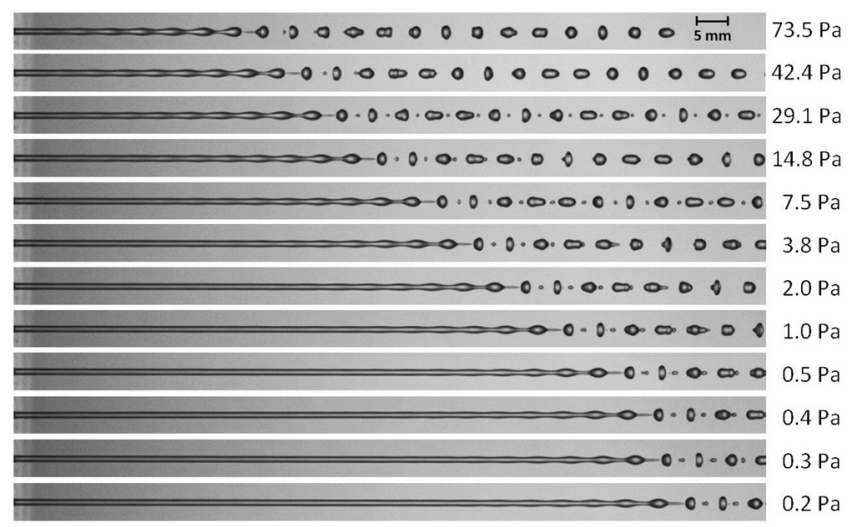

FIG. 2. Assemblage of representative frames from the high speed images for different pressure perturbations amplitudes. There is a transition from front pinching (top) to end pinching (bottom). 


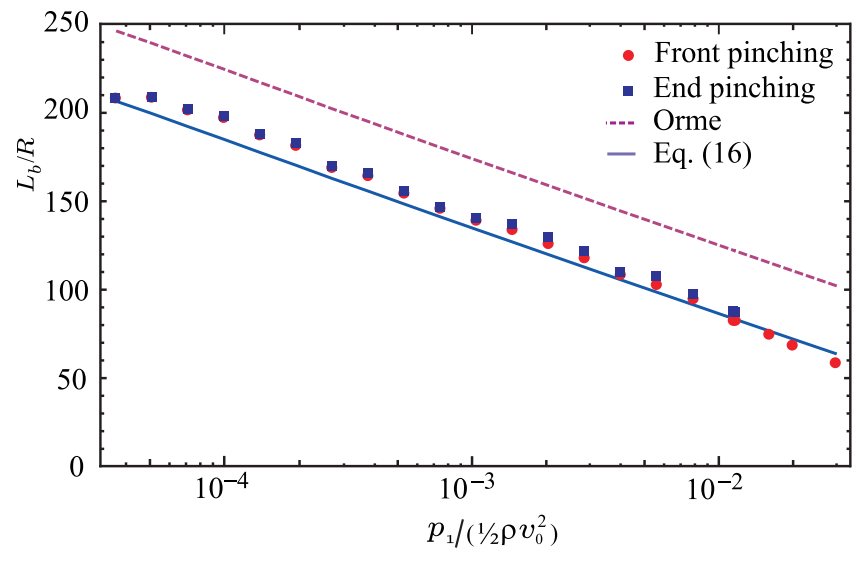

FIG. 3. Breakup lengths relative to the mean jet radii versus the amplitude of the pressure stimulation relative to the dynamic pressure at the jet exit. Breakup lengths are measured up to the front pinching or to the end pinching points. The lines are the two existing theoretical predictions for this experiment.

contraction coefficient $c_{\mathrm{c}}=0.795$ is obtained as well as the mean radii $R$ and mean Weber and Ohnesorge numbers for each breakup length ( $\mathrm{We} \simeq 50, \mathrm{Oh} \simeq 0.0056$ ). Finally, the entry correction factor is estimated as $K=1.90$ from (6) with $p_{0}=4320 \pm 30 \mathrm{~Pa}$ and assuming a null value for $l_{\mathrm{o}}$.

Fig. 3 shows the results of our experiments compared with the theoretical predictions. The estimated deviation in slope (related with the growth rate of the dominant capillary mode) is $2.5 \%$ and $4 \%$ in the initial amplitude of deformation, to be compared against $20 \%$ of Orme's theory. Although the acoustic impedances are roughly estimated in the theory, their small values in our experiment suggest that they are not the main sources of discrepancies. 1D numerical calculations in the spirit of those performed in Ref. 21 could be convenient to discern the accuracy of our linear approach. Another, not explored, possible explanation could be an unexpected decay of perturbations during the relaxation process of the initial velocity profile. In this case, 3D axisymmetric numerical simulations ${ }^{22}$ would be required to explore this further.

The theory presented in this work provides a simple and powerful tool that predicts the breakup length of pressure modulated capillary jets, confirmed by the experiments reported here. This theory could be employed to find both the optimal design of stimulation heads, particularly of the exit orifice, and the operating conditions to achieve prescribed breakup lengths. In the case of a piezoelectric device, the pressure modulation is not usually measured but predicted by a linear correlation with the voltage supply. Our transfer function should be able to validate that correlation. In addition, the modular construction of the transfer function will allow the incorporation of improvements such as (i) the hydrodynamics of the chamber and the exit orifice, (ii) the effect of the viscous relaxation region of the jet, and (iii) the subsequent jet evolution under different external conditions (coflow, electrification, etc.) with mere substitution of the corresponding dispersion relation.

This work was supported by the Spanish Government under Contract No. FIS2011-25161, by the Junta de Andalucía under Contract Nos. P09-FQM-4584 and P11FQM-7919, by the EPSRC-UK (Grant No. EP/H018913/1), the Royal Society and the John Fell Oxford University Press (OUP) Research Fund.

${ }^{1}$ J. Eggers and E. Villermaux, Rep. Prog. Phys. 71, 036601 (2008).

${ }^{2}$ E. R. Lee, Microdrop Generation (CRC Press LLC, 2003).

${ }^{3}$ J. R. Castrejon-Pita, W. R. S. Baxter, J. Morgan, S. Temple, G. D. Martin, and I. M. Hutchings, Atomization Sprays 23, 541 (2013).

${ }^{4}$ F. M. White, Fluid Mechanics (McGraw Hill, 2010).

${ }^{5}$ S. B. Segletes and W. P. Walters, Int. J. Impact Eng. 27, 561 (2002).

${ }^{6}$ J. Gavis and M. Modan, Phys. Fluids 10, 487 (1967).

${ }^{7}$ H. L. Langhaar, J. Appl. Mech. 9, A55 (1942).

${ }^{8}$ E. M. Sparrow, S. H. Lin, and T. S. Lundgren, Phys. Fluids 7, 338 (1964).

${ }^{9}$ C. A. Bruce, IBM J. Res. Dev. 20, 258 (1976).

${ }^{10}$ J. B. Keller, S. I. Rubinow, and Y. O. Tu, Phys. Fluids 16, 2052 (1973).

${ }^{11}$ J. Guerrero, H. González, and F. J. García, J. Fluid Mech. 702, 354 (2012).

${ }^{12}$ F. J. García and H. González, J. Fluid Mech. 602, 81 (2008).

${ }^{13}$ L. Rayleigh, Philos. Mag. 34, 145 (1892).

${ }^{14}$ S. Chandrasekhar, Hydrodynamic and Hydromagnetic Stability (Dover, New York, 1961).

${ }^{15}$ F. J. García and A. Castellanos, Phys. Fluids 6, 2676 (1994).

${ }^{16}$ H. González and F. J. García, J. Fluid Mech. 619, 179 (2009).

${ }^{17}$ M. E. Orme, "A new approach to the study of capillary stream break-up," Ph.D. dissertation (University of Southern California, 1989).

${ }^{18}$ S. A. Curry and H. Portig, IBM J. Res. Dev. 21, 10 (1977).

${ }^{19}$ J. M. Schneider, N. R. Lindblad, C. D. Hendricks, and J. M. Crowley, J. Appl. Phys. 38, 2599 (1967).

${ }^{20}$ J. L. Dressler, Phys. Fluids 10, 2212 (1998).

${ }^{21}$ B. Ambravaneswaran, H. J. Subramani, S. D. Phillips, and O. A. Basaran, Phys. Rev. Lett. 93, 034501 (2004).

${ }^{22}$ B. Ambravaneswaran, E. D. Wilkes, and O. A. Basaran, Phys. Fluids 14, 2606 (2002). 